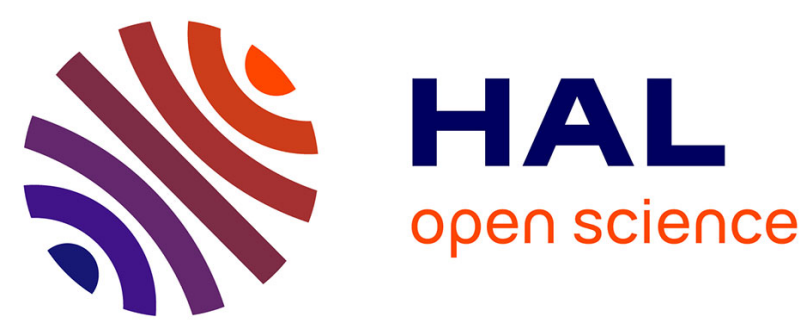

\title{
Towards a Spatial Semantics to Analyze the Visual Dynamics of the Pedestrian Mobility in the Urban Fabric
}

Thomas Leduc, Francis Miguet, Vincent Tourre, Philippe Woloszyn

\section{- To cite this version:}

Thomas Leduc, Francis Miguet, Vincent Tourre, Philippe Woloszyn. Towards a Spatial Semantics to Analyze the Visual Dynamics of the Pedestrian Mobility in the Urban Fabric. Marco Painho; Maribel Yasmina Santos; Hardy Pundt. Geospatial Thinking, Springer Berlin Heidelberg, pp.237-257, 2010, 978-3-642-12325-2. 10.1007/978-3-642-12326-9_13 . hal-01347345

\section{HAL Id: hal-01347345 \\ https://hal.science/hal-01347345}

Submitted on 20 Jul 2016

HAL is a multi-disciplinary open access archive for the deposit and dissemination of scientific research documents, whether they are published or not. The documents may come from teaching and research institutions in France or abroad, or from public or private research centers.
L'archive ouverte pluridisciplinaire HAL, est destinée au dépôt et à la diffusion de documents scientifiques de niveau recherche, publiés ou non, émanant des établissements d'enseignement et de recherche français ou étrangers, des laboratoires publics ou privés. 


\title{
Towards a spatial semantics to analyze the visual dynamics of the pedestrian mobility in the urban fabric
}

\author{
Thomas Leduc $^{1}$, Francis Miguet ${ }^{1}$, Vincent Tourre ${ }^{1,2}$, Philippe Woloszyn ${ }^{3}$ \\ ${ }^{1}$ CERMA laboratory UMR CNRS 1563, 6 quai François Mitterrand, BP \\ 16202, 44262 Nantes cedex 2, France - \{thomas.leduc, francis.miguet, \\ vincent.tourre\}@cerma.archi.fr \\ ${ }^{2}$ Ecole Centrale de Nantes, rue de la Noë, BP 92101, 44321 Nantes ce- \\ dex 3, France \\ ${ }^{3}$ RESO laboratory, ESO, Maison de la Recherche en Sciences Sociales, \\ Université de Haute Bretagne - Rennes II, place du recteur Henri Le Moal, \\ CS 24307, 35043 Rennes cedex, France - philippe.woloszyn@univ- \\ rennes2.fr
}

\begin{abstract}
The aim of this paper is to evaluate the impact of visual ambiences (visualscape) onto an urban pedestrian pathway. This work takes place within the interdisciplinary research project called Ambioflux, dealing with the sustainable mobility issues in the city. We add to the spatial semantic layer GDMS (Generic Datasource Management System) an innovative method based on partial isovists fields in order to compute the visibility of the pedestrian all along his pathway. This method allows "concatenating" several partial visibility polygons in order to represents the visual perception of the pedestrian.

After a brief overview of the visibility analysis context, we justify the need of a specific semantic tool to develop the type of dynamics visual analysis we focus on. The remainder of this paper is dedicated to the methodology of the mobile pedestrian pathway's visual fingerprint characterization using the spatial formalism already described. At last, we present a use case based on a real city tour so as to identify the best rotation's direction from the visual perception point of view.
\end{abstract}




\section{Introduction}

The Ambioflux's interdisciplinary project deals with the urban ambiences in the context of the city re-qualification. The aim of this project is to focus on both environmental and eco-systemic aspects of the study but also on its psychological and anthropological characteristics.

In the context of this research project, the objective of the current task is to evaluate, so as to characterize, the impact of visual ambiences (visualscape) onto an urban pedestrian pathway using and extending the spatial semantic layer called GDMS (Generic Datasource Management System).

(Zacharias, 2001) wrote: the sensory experience of the environment may dominate the information field and hold sway over many of our actions. We personally adhere to (Piombini, 2006)'s simple hypothesis: visualscape plays an important role in the pedestrian's perception of the surrounding urban environment. Thus, the optimization of the visualscape's assessment is a good solution to enhance walking practices.

After a brief overview of the visibility analysis context, we justify the need of a specific semantic tool to develop the type of dynamics visual analysis we focus on. The remainder of this paper is dedicated to the methodology of the mobile pedestrian pathway's visual fingerprint characterization using the spatial formalism already described. At last, we present a use case based on a real city tour so as to identify the best rotation's direction from the visual perception point of view.

\section{Overview of visibility analysis}

In (Gibson, 1979), an ecological theory of visual perception, based on optic flow patterns, has been defined. In this theory, real movement plays a vital part in the perception process. The three key ideas of it are: optic array, textured gradients, and affordance. The optic array corresponds to the set of all visual information (about the layout of objects in space) reaching the eye. It is a set of nested solid angles corresponding to surface elements in the environment. The textured gradient provides information about the characteristics (distance, speed, etc.) of the perceived environment. This mechanism requires almost a small signal processing by the cognitive system. At last, the affordance implies that a particular meaning is enclosed in each visual information: the potential use of an object is directly perceivable (e.g. a chair 'affords' sitting). 
In the 1970s, two main approaches emerge in the visibility analysis context: the concept of viewshed in terrain and landscape analysis and the concept of isovist in architecture and urban space.

The viewshed analysis is a traditional way of analyzing a visibility field. It is defined as the part of terrain visible from a viewpoint, and is basically applied to the landscape with terrain and topographic differentiation (Lynch, 1976). As noticed in (Yang, 2007), viewshed analysis in GIS is rarely applied to urban settings because the operation is based on raster data or TIN (triangular irregular network) data structure, which have problems of accuracy in representing complex geometry of urban form.

(Benedikt, 1979) defines an isovist as "the set of all points visible from a given vantage point in space and with respect to an environment". This concept has been previously introduced in (Tandy, 1967). As mentioned in one of (Benedikt, 1979) footnotes, "readers familiar with Gibson's work will see the affinity of isovists with [ambient] optic arrays". The isovist is simply this optic array, with the wavelength and intensity information omitted (Benedikt and Burnham, 1985). To sum up, an isovist is usually a $2 \mathrm{D}$ bounded polygon which is a useful tool to define the open space concept. From a morphological point of view, open spaces are usually defined as the empty space, the void, between the surrounding buildings. However, although these open spaces are not material components of the physical world, they can be conceived as part and parcel of our urban heritage (Teller, 2003). (Batty, 2001) puts the emphasis on the fundamental motivations of conducting visibility analysis research. He noticed that the key questions "how far can we see", "how much can we see", and "how much space is enclosed" are relevant to develop good urban design.

The Gibsonian panoramic visual world can be seen as a set of temporal series of excitations (sort of visual snapshots) one might experience moving about inside the urban fabric. The pedestrian faces urban morphology in the context of its natural movements. As written in (Yang, 2007), human perception is influenced and to a certain extent can be manipulated by reconfiguring physical urban morphology, under particular ambients constraints (Woloszyn, 2002).

Several different spatial analysis methods have been defined to study the plenum conception (Couclelis, 1992) of urban space: isovist fields (Benedikt, 1979; Batty, 2001), space syntax (Hillier and Hanson, 1984), visibility graphs (Turner et al., 2001), agent-based approach (Batty and Jiang, 1999), convex partitions (Peponis et al., 1997), sky opening maps (Teller, 2003; Sarradin et al., 2007) and ambianscape formalization (Woloszyn et al., 2000). Some of them - agent-based and visibility ones - are based on a regular grid medium on which all subsequent analysis is developed, that's the reason why we have dismissed them. Some other assumes that urban 
open spaces can be uniquely partitioned into convex sets that meet some criteria of minimality in number. The scientific community is divided over this issue, that's why we did not focus on convex partitions. At last, according to (Yang, 2007), the Teller's sky opening indicator, which requires double projections of its spherical surface for its visual indicator, makes the original 3D spherical surface distorted and deformed, and easily induces incorrect calculation.

For all these reasons, we have decided to focus on the well-known 2D isovist fields (vector based approach) method. Indeed, (Hillier and Hanson, 1984) noticed "human space is in fact full of strategies [...] to reduce the three-dimensional structures to the two dimensions in which human beings move and order space". Essentially, isovists describe local geometrical properties of spaces with respect to individual observation points and weight all the possible view directions equally. An isovist is a $2 \mathrm{D}$ horizontal slice of pedestrian's surrounding space.

For the specific analysis of motion trajectories, (Meilinger, 2009) processes view-specific partial isovists. Partial isovists consider only a restricted part of the theoretically available visual field (for example, $90^{\circ}$ instead of $360^{\circ}$ ). They correspond better to the restrictions of the human visual apparatus. To reproduce the dynamic qualities of the Gibsonian visual world that takes into account not only the bifocal vision but also the relatively free movement of the head and shoulders (Teller, 2003), one may consider the partial isovist angle as an input parameter of the parametric model so as to produce range of results.

\section{Why do we need to merge a GIS\& $T^{1}$ semantic tool and visibility analysis?}

What seems obvious with all previous models is the lack of wide scale simulations on motion approach. Indeed, the mathematical combination of a set of isovists results in a multitude of heterogeneous geometric shapes that needs to be analyzed. It seems important to address it regarding the most appropriate scientific tool. Thus we have decided to focus on one of the Association of American Geographers (AAG) reference studies. The Geographic Information Science and Technology Body of Knowledge ${ }^{2}$ (BoK) is a community-developed inventory of the knowledge and skills that define the GIS\&T field. In order to achieve the analysis of the visibil-

\footnotetext{
${ }^{1}$ GIS\&T: Geographic Information Science and Technology.

${ }^{2}$ http://aag.org/bok/ (valid on Jan. 6th 2010).
} 
ity all along a pedestrian pathway, we have clearly identified several involved sub-domains of this BoK such as the following:

- in term of analytical methods:

- query operations and query languages (spatial queries),

- geometric measures (distances and lengths, areas, etc.),

- basic analytical operations (buffers),

- basic analytical methods (spatial interaction),

- in term of cartography and visualization (data considerations, principles of map design),

- in term of design aspects

- analysis design (coupling scientific model with GIS),

- application design (workflow analysis and design),

- in term of conceptual foundations (fields in space and time, metrical relationship),

- in term of data modeling (classic vector data model),

- in term of geocomputation (simulation modeling).

This observation has already been implicitly made even if it does not seem to have yet been translated into such an inventory. So, GIS-based visibility analyses are nowadays quite a common approach. Indeed, an urban pedestrian pathway and a visualscape are both data that include a spatial component. What is required here is some tool able to process these spatial data using: on one hand the table-oriented programming paradigm (for its table-friendly syntax, its fundamental and consistent collection operations, and its easiness of understanding) and, on the other hand, batch processing with parametric ability and procedural extension. We pretend that the use of a spatial SQL with semantics ability is essential to perform such an objective. That is the reason why we need to take benefits from the GDMS specific layer, aside the features of robustness, scalability, and easy to use main characteristics.

GDMS (Generic Datasource Management System) is related with a similar layer, called GDBMS (Anguix and Carrion, 2005), used in the gvSIG project to manage the alphanumeric data access. The main limitation of the original GDBMS layer is that it can only be used for alphanumeric purposes. The GDMS contribution is a general refactoring that mainly adds spatial functionalities and a more powerful SQL processor. It has been defined and implemented in the context of the OrbisGIS project, and extended in its recent GearScape customization (with a sort of procedural ability). The relevance of this layer for spatial processing has already been exhibited by (Leduc et al., 2009a, 2009b). 
The main objectives of GDMS are to provide to the user not only a simple and powerful API but also a spatial SQL derived language. Moreover, as an intermediate layer between the user and the information source, GDMS intends to reduce the coupling between the processes and the specificities of each underlying format. As a consequence, former work may easily be reused in a much larger set of scenarios. The learning curve is consequently even simpler.

With GDMS, the idea is to move all problems of interoperability across data repositories, but also about the SQL semantics by developing a highly flexible, portable and standards compliant tool to build SQL queries. GDMS provides a SQL processor that lets the execution of the common Data Manipulation Language statements against any source mapped by a driver. To avoid introducing a new grammar, GDMS fully preserves the SQL-92 grammar and adds to this standard geometric concepts and spatial functions as in OGC simple features SQL specification. As an analogy to spatial SQL for R-DBMS, GDMS provides an extended SQL query language on heterogeneous data types.

It is the main purpose of GDMS to improve data creation and sharing. As in a Spatial Data Infrastructure (SDI) the consumption of data is as important as the production and sharing of data, the SQL processor in GDMS allows data feedback as if they were new data sources (materialized views). This means that the result of the SQL queries can easily be integrated into the SDI as a new data source. Those data will be ready to be used by further SQL statements as any other existing data source.

GDMS allows an extension to the semantics of the SQL language in terms of functions and custom queries. The functions and custom queries are artifacts that contain the implementation of some operations on the data and can be reused just by referencing their name into a SQL statement. In this way, some user can implement a buffer operation and other can reuse it just by calling $S T$ Buffer() in a SQL query:

SELECT ST_Buffer(the_geom, 20) FROM mydata;

\section{Methodology}

\subsection{Prerequisites}

We want our model to rely on "classical" data of vector type so as to let it be easily and widely repeatable. That is why, in the following use case, we present a simulation that is only based on both a polygonal buildings layer 
and a linear pathway layer. Though, it is obvious that it could be enriched with several other layers such as urban vegetation or street furniture. In the same manner than the buildings layer, they would be treated as visual masks (the simpler is probably to union all the mask layers in a preprocessing phase).

What needs to be remembered here is that a pedestrian walk in the urban fabric is fundamentally a time-line process. To simplify the model, we have decided to adopt a discrete time approach of the perception mechanism: a slice-time has to be set so as to sequence each pathway. The mean speed of the pedestrian is stable, at each time tick, he reaches a new following position. The continuous linear pathway is thus transformed into a set of ordered punctual equidistant positions. In each of these positions, an isovist is computed and the corresponding polygon is then added to the global isovists field.

As written in the visibility analysis overview section, for the specific analysis of motion trajectories, (Meilinger, 2009) processes view-specific partial isovists. To reproduce the dynamic qualities of the Gibsonian visual world, we have decided to consider the partial isovist angle as an input parameter of our parametric model so as to produce range of results. To illustrate the topic in the following use case, five distinct aperture angles are mentioned: $30^{\circ}, 60^{\circ}, 90^{\circ}, 140^{\circ}$ and $200^{\circ}$. Even if none of them are clearly identified in both the visual perception theory and the cognitive psychology science, we may admit that a visual object is less avoidable and much stronger in term of continual awareness, if it is located in front of us (small aperture angle) than far left- or right-sided.

We are aware that such preconditions are extremely simplistic and thus unadapted to model the visual perception of a pedestrian immersed in a composite visualscape. The aim here is to simplify so as to be able to simulate. In the conclusion we will suggest several possible enhancements.

As mentioned in the visibility analysis overview, we clearly need a model able to produce a directional visibility analysis that take also into account visual dynamics all along the pathway (motion approach).

\subsection{Sequence of spatial processing}

The simplified sequence diagram presented in Fig. 1, sums up the main phases of the visual dynamics analysis method we have developed. The global process is composed of the following different phases and sub phases: 
1. sample the continuous pathway in both directions so as to obtain a set of equidistant punctual positions and, for each of them, the corresponding local speed vector,

2. perform the isovists field computation. This step is achieved using a dedicate development that makes a strong use of the GDMS efficient spatial index implementation. It is a vector based process much more accurate than a raster one;

3. loop on the aperture angle values (parametric study) and, for each of them:

- evaluate the visual cones in each punctual position (according to the speed vector direction) and calculate the intersection with the corresponding isovist so as to produce a partial isovist,

- compute the weighted coverage of the resulting partial isovists field. The aim is here to produce, using a dedicated polygonal coverage algorithm, a partition of the input (partial) isovists fields. That is, a division of it into non-overlapping and non-empty subsets that cover all of it. These parts are collectively exhaustive and mutually exclusive. 


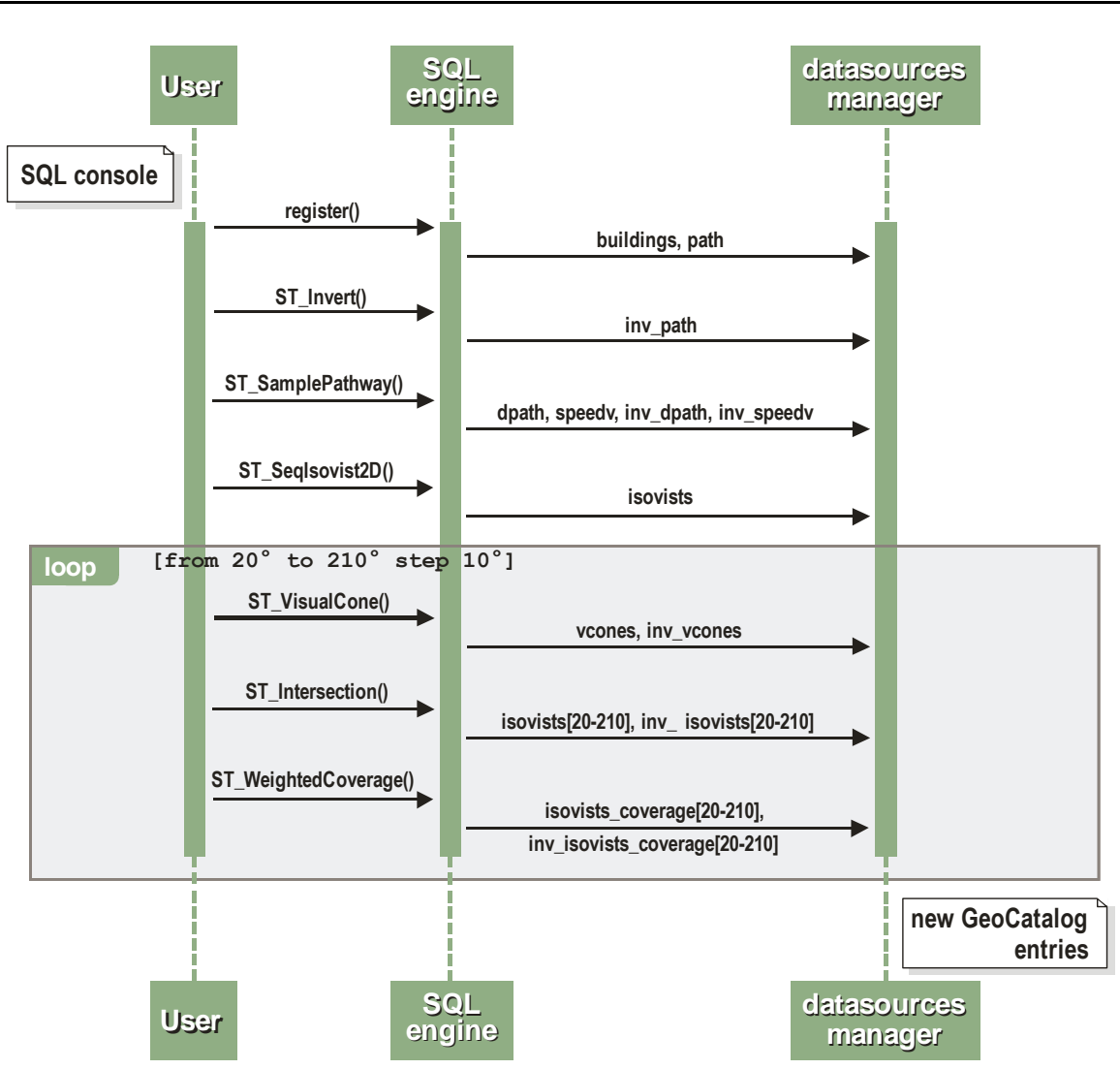

Fig. 1. Simplified sequence diagram of the spatial processes involved in the visual analysis. The aim is here first to produce a set of partial isovists fields and then to produce a weighted coverage of the global visible area

\subsection{Post-processing}

The objectives of the processing phases presented above are to produce both a set of partial isovists fields (for different values of aperture angles) and a set of weighted coverage of all the corresponding global visible areas. The weighted coverage process is illustrated in Fig. 2. It consists in a partition of the geometry union of all input polygons. In this output partition, each item is "weighted" with a counter equals to the number of input polygons that contain it. Producing such a weighted coverage map is useful to analyze the urban areas that are promoted from a temporal point of view (that is, all those the pedestrian is able to look upon for a long time) and all those that are neglected (almost outside his field of vision). 
Another post-process consists in producing indicators to characterize each isovists field. So, we need to identify a few sets of relevant visual indicators. The already defined ones often correspond to "complete" isovists fields (Benedikt, 1979; Batty, 2001). We must keep in mind the fact that we deal with partial isovists fields (fundamentally asymmetric). The translation into formal descriptors is not a so easy task because the meaning and relevance of descriptors are difficult to estimate a priori. In the following use case, we will comment two simple scalar indicators. The first one is the polygonal area of each isovist, and the second corresponds to the number of edges of each isovist.

\section{Focus on the weighted coverage algorithm}

We want to thank M. Davis, primary designer and developer of the JTS Topology Suite ${ }^{3}$ library, for his decisive help in designing it. As written before, it consists in a partition of the geometry union of all input polygons where each output polygon is "weighted" with a counter equals to the number of input polygons that contain it. This memory and CPU consuming process is divided into the 4 following phases:

1. extract the linework (boundaries) of the input polygonal partial isovists,

2. node the linework. The JTS UnaryUnionOp is a convenient way to do this (Union on a set of LineStrings has the effect of noding them);

3. pass the noded linework into JTS Polygonizer and polygonize it,

4. for each resultant polygon, determine an interior point and then find the source polygons which contain this point so as to weight it (using spatial index and PreparedGeometry speed the process up).

\footnotetext{
${ }^{3}$ http://tsusiatsoftware.net/jts/main.html (valid on Jan. 6th 2010).
} 

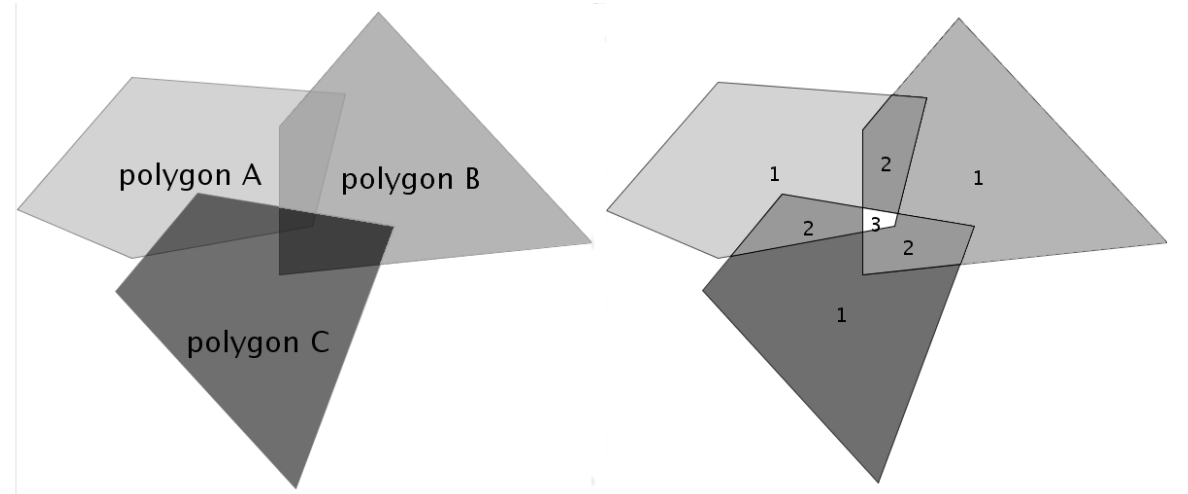

Fig. 2. Illustration of the weighted coverage process on a set of three overlapping polygons. The result is a partition of the geometry union of all input polygons. In this output partition, each item is "weighted" with a counter equals to the number of input polygons that contain it

\section{Use case}

In order to exhibit the relevance of our model and its potentialities, we have decided to apply it to a pedestrian pathway called "parcours confort" proposed ${ }^{4}$ by the Nantes Métropole Tourist Office (NMTO). Nantes is a west-coast located city in France. This tour, accessible to all (even for people in a wheelchair), proposes to those who have some time to spend wandering in Nantes a discovery of the historical city centre from medieval to $19^{\text {th }}$ century morphologies (see Figures 3 and 4). This 1590 meters long tour starts from the tramway central station Commerce and run through:

- the medieval district called quartier du Bouffay,

- the Cours des 50 otages main street, named in memory of the fifty hostages who were executed during the Second World War. This boulevard has been redesigned in 1990 by the Italian architect Italo Rota;

- the Place Royale, which embeds a 19th century fountain representing the city of Nantes with the Loire River sitting on her feet,

- the Passage Pommeray, the only multilevel covered street in Europe, containing a staircase bordered by statues.

\footnotetext{
${ }^{4}$ This tour is available on-line on the website: http://www.nantes-tourisme.com/ (valid on Jan. $6^{\text {th }} 2010$ ).
} 
Our goal is to compare, in term of the visual perception of a pedestrian, the former NMTO tour in both clockwise (CW) and counter-clockwise $(\mathrm{CCW})$ directions.

\subsection{Comparison of the global visual coverage rate of both directions on the NMTO tour}

We assume that this 1590 meters long tour has been divided into 983 punctual equidistant positions or time steps. The distance between each of these positions is equal to 1.6 meter (time-slice between two successive "snapshots" is approximately equals to 1.5 second for a walking speed of about $4 \mathrm{~km} / \mathrm{h}$ ). In each position, several isovists have been computed according to the variation of the aperture angle.

Fig. 3 presents a weighted coverage of each partial isovists field (they both share the same aperture angle equals to $60^{\circ}$ ). The left one corresponds to the counter-clockwise oriented pathway, while the right one corresponds to the clockwise oriented pathway. One may notice that the visual aspect of those both maps are rather different: visual-walk is a one-way process.

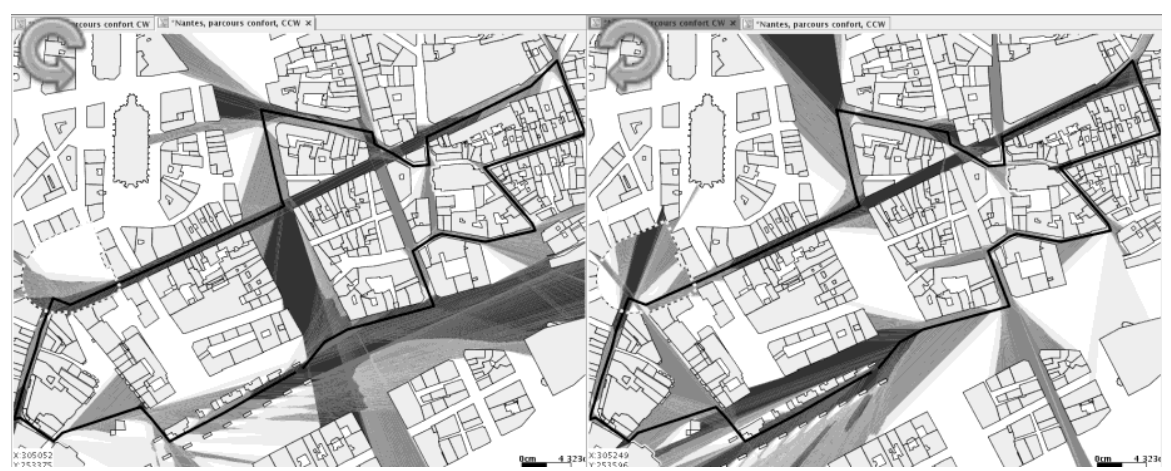

Fig. 3. Comparison of two partial isovists fields (with an aperture angle equals to $60^{\circ}$ in both cases) of the same NMTO tour in the historical city centre (black line). On the left hand side, pathway is counter-clockwise (CCW) oriented while, on the right hand side, it is clockwise $(\mathrm{CW})$ oriented. Shapes underlined correspond to some specific locations that will be developed later in this use case

The three different shades of grey presented in the Fig. 3 correspond to an interval classification:

- the light grey corresponds to an interval from $0 \%$ to $1 \%$,

- the medium grey corresponds to an interval from $1 \%$ to $5 \%$,

- and the dark grey corresponds to an interval from $5 \%$ to $22 \%$. 
These percent values correspond, for a given polygonal area, to the time it has been seen (with an aperture angle equals to $60^{\circ}$ ) by a given pedestrian all along the 983 punctual positions.

In the Fig. 4, we identify the indices of the punctual positions so as to be able to analyze the results we present in both the Fig. 5 and the Fig. 6.

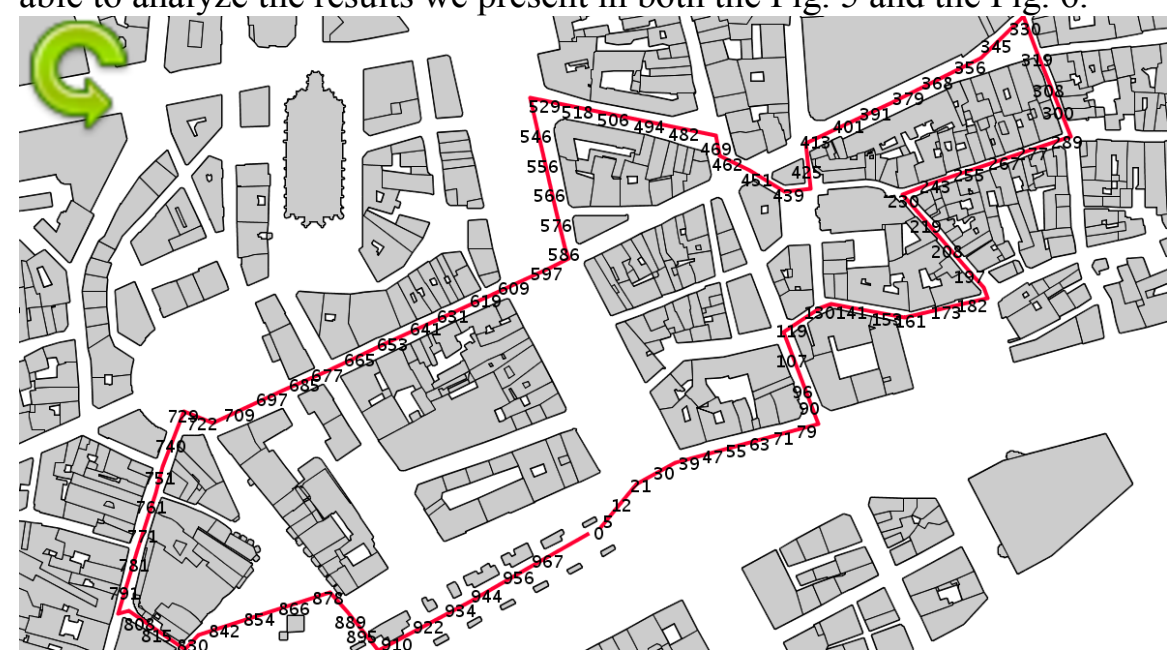

Fig. 4. Screenshot of both buildings and pedestrian pathway layers. As may be noticed, the continuous pathway is sampled into 983 punctual positions so as to let us perform the isovists fields computations

The Fig. 5 and Fig. 6 illustrate the ability of our tool to produce sort of signatures of the partial isovists field (with an aperture angle always equals to $60^{\circ}$ ) according to the evolution of a given scalar indicator all along each pathway. Aim is here to compare, for a given pedestrian punctual position, the area of the partial isovist (in both directions) and its number of edges (in both directions also). 


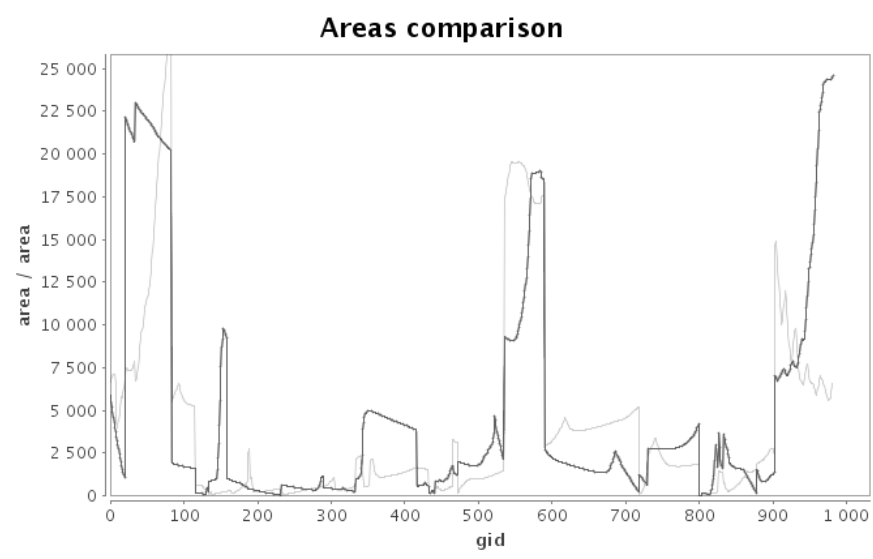

Fig. 5. This line chart represents the evolutions of the area indicator all along each pathway (CCW one is represented by the dark grey line and $\mathrm{CW}$ one is represented by the light grey line)

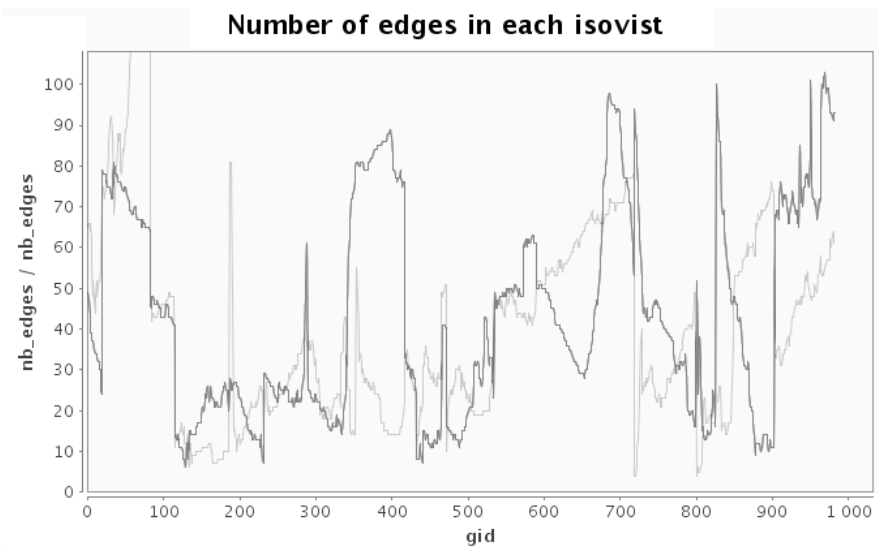

Fig. 6. This line chart represents the evolutions of the number of edges indicator all along each pathway $(\mathrm{CCW}$ one is represented by the dark grey line and $\mathrm{CW}$ one is represented by the light grey line)

One may notice that around position \#425 (Sainte Croix church), the $\mathrm{CCW}$ number of edges (in dark grey) is 3 times greater than the corresponding CW number of edges (in light grey). As a consequence, the pedestrian isovist is quite fuzzy in the CCW direction.

Concerning the punctual position around \#720 the visual area of the CW oriented pathway (in light grey) is two times greater than the visual area of the CCW oriented pathway (in dark grey). Indeed, Saint Nicolas church is only visible, at this position, from the $\mathrm{CW}$ oriented tour! 


\subsection{Zoom in some specific areas so as to show different comparison methods}

In this section, we will compare different methods to analyze the perceived environment. Each of them will be applied to a specific urban object in an adapted context.

\section{Visible surfaces ratio of an urban square: the example of the Place Royale}

The surface ratio is the visible surface in the studied tour over the total surface of the Place Royale square (see Fig. 7). In this figure, the light grey polygons correspond to partial isovists of aperture angle equals to $30^{\circ}$. The medium grey polygons (that contain also the light grey one) correspond to partial isovists of aperture angle equals to $60^{\circ}$ and, at last, the dark grey polygons correspond to partial isovists of aperture angle equals to $90^{\circ}$. One may notice than this square and especially its nice central fountain is best seen with the $\mathrm{CW}$ tour.

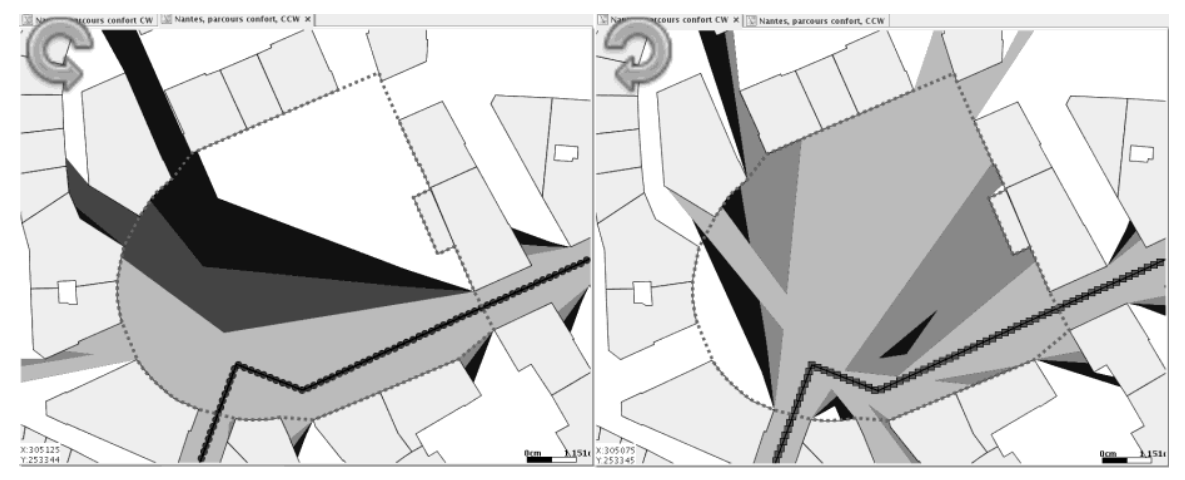

Fig. 7. Amount of visible areas for each pathway. The CW tour (on the right) covers obviously much more the square than the $\mathrm{CCW}$ one (on the left). In those both screenshots, light grey polygons correspond to a partial isovist field of $30^{\circ}$, medium grey ones to $60^{\circ}$ partial isovists fields and dark grey ones to $90^{\circ}$ partial isovists fields

As an illustration, we have computed coverage ratios for each of the aperture angles:

- for the CW tour: $64.7 \%$ is visible with an isovist of $30^{\circ}, 88.7 \%$ with an isovist of $60^{\circ}$, and $93.7 \%$ with an isovist $90^{\circ}$;

- for CCW tour: $36.6 \%$ is visible with an isovist of $30^{\circ}, 52.4 \%$ with an isovist of $60^{\circ}$, and $67.7 \%$ with an isovist $90^{\circ}$. 


\section{Visibility of a specific valuable urban object: the example of the Saint Nicolas church}

In the Fig. 8, we demonstrate that the front of Saint Nicolas church is only seen during 2 time steps (from positions \#727 to \#728) over 983 with an aperture angle greater of equal than $200^{\circ}$. Those both isovists give the pedestrian the possibility to see only $6.89 \%$ of the total façade of this church.

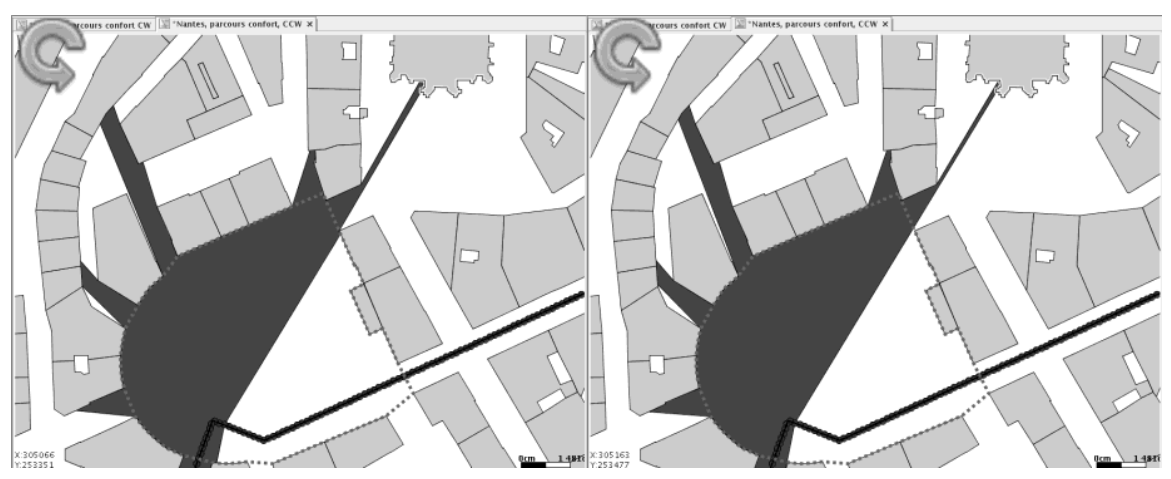

Fig. 8. Isovist for positions \#727 (on the left) and \#728 (on the right) for the CCW tour

Comparing Fig. 8 and Fig. 9, it is obvious that Saint Nicolas church is best seen (2.5 more length) and for a longer time (12 times more) in the $\mathrm{CW}$ tour.

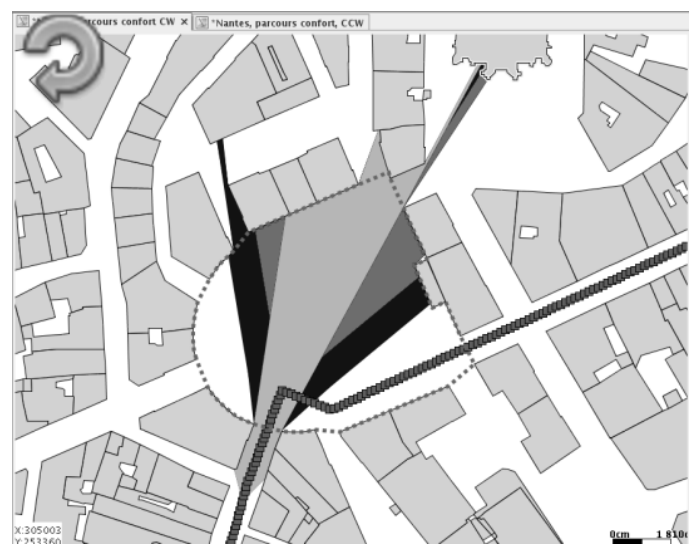

Fig. 9. Partial isovists fields (aperture angles from $30^{\circ}$ - light grey polygon, to $90^{\circ}$ - dark grey polygon) from position \#230 to position \#253 in the CW oriented tour 


\section{Visibility of a specific valuable urban front: the example of the Sainte Croix church}

In the specific case of Sainte Croix church, in the $\mathrm{CCW}$ oriented tour (on the left) the pedestrian is once again badly positioned to see the front of the monument (see Fig. 10).

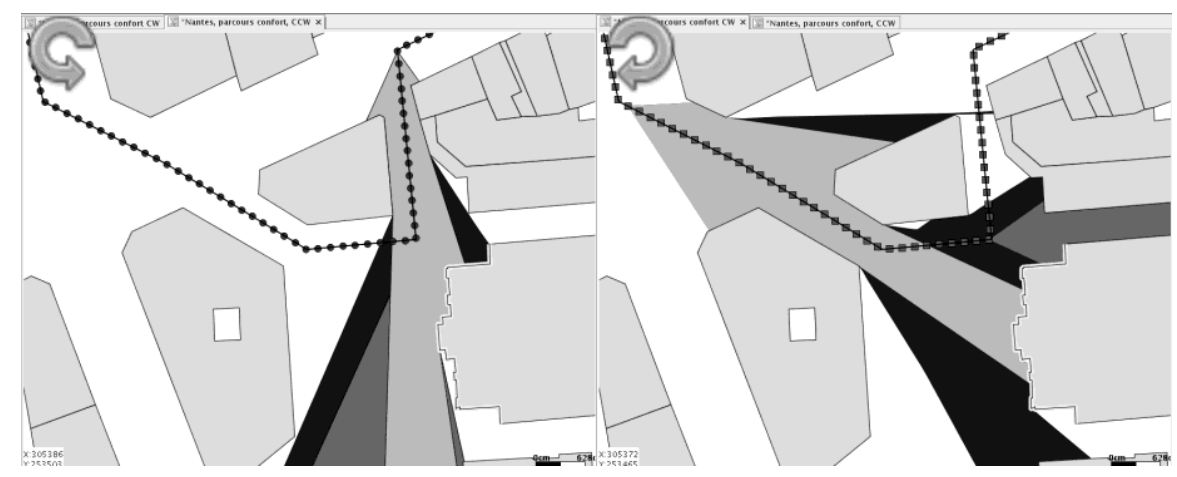

Fig. 10. The $\mathrm{CCW}$ oriented tour (on the left) gives the pedestrian the possibility to see at most $50 \%$ of the front of this nice building. On the contrary, with the same aperture angle, in the $\mathrm{CW}$ oriented tour, the front of Sainte Croix church is much more seen. Moreover, in the $\mathrm{CW}$ tour, the incidence angle is far better $\left(40^{\circ} \mathrm{ac}-\right.$ cording to the normal to the façade). In those both screenshots, the color code corresponds to some different notions. Indeed, the light grey polygon is the first isovist of the pedestrian walk that contains part of the façade (positions \#417 in the $\mathrm{CCW}$ tour and \#518 in the CW one). The same way, the medium grey polygon is the last isovist that contains part of the façade (positions \#432 in the CCW tour and \#550 in the $\mathrm{CW}$ one). By contrast, the dark grey polygons are partial isovists fields from position \#417 to \#432 in the CCW tour and from position \#518 to $\# 550$ in the $\mathrm{CW}$ one

\section{Visibility and distance: the example of the place de Sarajevo square}

In this last example, it is once again obvious that the front of the old stock exchange is much more visible in the $\mathrm{CW}$ oriented tour than in the $\mathrm{CCW}$ one (see Fig. 11).

In conclusion of the use case, these results clearly show that some of the most important buildings are not well seen in the CCW tour and that the CW tour allows a better discovery of the city. A good feedback of the person in charge of the tourist tours, encourage us to apply this method to analyze some other city tours presented on the Nantes Métropole Tourist Office website. 


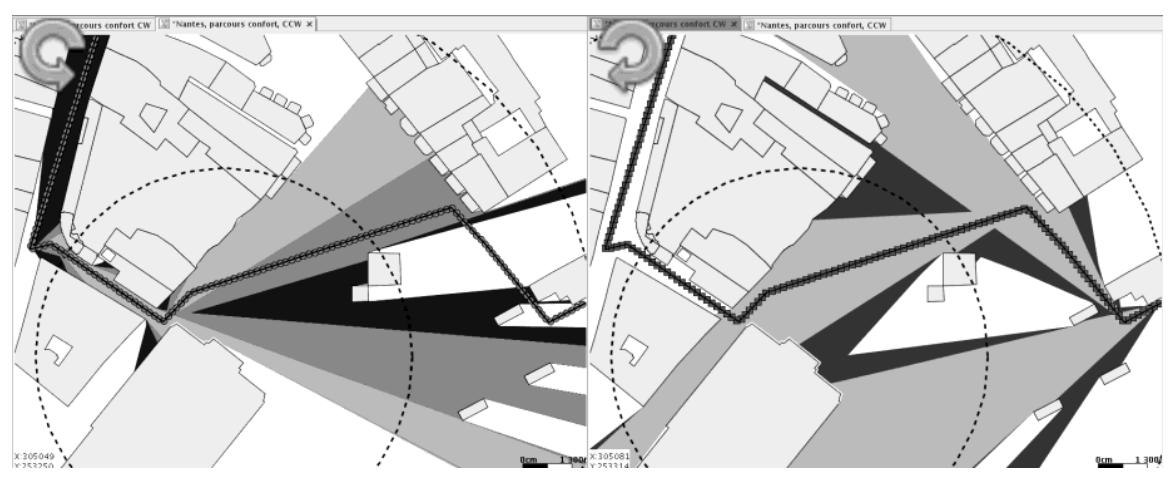

Fig. 11. In the CCW oriented tour (on the left), even with an aperture angle equals to $140^{\circ}$, less than half of the front of this building is visible. In the $\mathrm{CW}$ oriented tour (on the right), a partial isovist with an aperture angle equals to $60^{\circ}$ is enough to see it fully. Moreover, it is fully visible with a distance less than 90 meters (and a comfortable incident angle). The color code of this figure is once again specific: light grey polygons correspond to $30^{\circ}$ partial isovists fields, medium grey polygons correspond to $60^{\circ}$ partial isovists fields and dark grey ones to $140^{\circ}$ partial isovists fields

\section{Conclusion and outlook}

The use case that has been presented in this paper is relative to Nantes historical center. It is however obvious that the methodology we described is transposable to any other pedestrian pathway in an urban fabric and, more generally but with some limits, to any journey through a built environment independently from the means of transportation. As an example, in a public transportation such as a bus, a trolley bus or a tramway, passenger's partial isovist main direction is often perpendicular to the motion one. What differs the most is probably the distance between two consecutive snapshots; indeed it depends on the displacement's speed.

Another practical application case is relative to the visual impact of a new structure, such as a building, on its surrounding open space. What kind of visual masks does it involves? Does it hide significantly some interesting view? It is also probably a useful tool in the decision making process for the positioning of urban road signs, etc.

Concerning the decision we took to couple GIS\&T and visibility analysis, this study is another proof of the relevance of the GDMS layer in term 
of spatial knowledge enhancement. Much more than a syntax with spatial abilities, this layer provides an extensible engine and a set of tools that let us combine the physical features perceived so as to infer the pedestrian feeling and therefore behavior. Empowered with efficient semantics ability one may admit that a new GIS dedicated to the characterization of a visualwalk in the urban fabric has thus been developed.

Regarding next steps of our study, we aim to focus on some specific and relevant indicators (such as jaggedness, radial variance and radial skew, convex deficiency... (Stamps, 2005)) so as to characterize partial isovists fields. The visualscape indicators we plan to implement have mainly to integrate the psychophysical dimension of the perception process (Woloszyn, 2002). At last, we have decided to focus on a $2.5 \mathrm{D}$ implementation of this model (coupling it with the ones presented in (Morello and Rati, 2009) and (Yang et al., 2007)) so as to take surrounding buildings, with their elevation component, into account for enhanced mask effects computation.

\section{Acknowledgements}

The AMBIOFLUX project was funded by CNRS and the French MEEDM Ministry under PIRVE's (Programme Interdisciplinaire de Recherche Ville et Environnement) contract \#1004. Part of the GearScape developments is funded by the AMBIOFLUX project.

Special thank to Fernando GONZÁLEZ CORTÉS (Spain) for all the developments performed on the GearScape platform.

\section{References}

Anguix, A. and Carrion, G. (2005). gvSIG: Open Source Solutions in spatial technologies. In GIS Planet, Estoril, Portugal.

Batty M (2001). Exploring isovist fields: space and shape in architectural and urban morphology. Planning and design: Environment and planning B, 28(1):123-150.

Batty M and Jiang B (1999). Multi-agent simulation : new approaches to exploring space-time dynamics in GIS. CASA Working Papers Series, London, UK, (10): 25 .

Benedikt ML (1979). To take hold of space: isovists and isovist fields. Environment and Planning B: Planning and Design, 6(1):47-65. 
Benedikt ML and Burnham CA (1985). Perceiving architectural space: From optic arrays to isovists. In Persistence and Change, pages 103-114. edited by WH Warren and RE Shaw. Hillsdale, NJ: Lawrence Erlbaum Associates.

Couclelis H (1992). People Manipulate Objects (but Cultivate Fields): Beyond the Raster-Vector Debate in GIS. In Frank AU, Campari I, and Formentini U, editors, Theories and Methods of Spatio-Temporal Reasoning in Geographic Space, International Conference GIS - From Space to Territory: Theories and Methods of Spatio-Temporal Reasoning, Lecture Notes in Computer Science, pages 65-77, Pisa, Italy. Springer.

Gibson JJ (1979). The Ecological Approach to Visual Perception. Boston: Houghton Mifflin.

Hillier B and Hanson J (1984). The Social Logic of Space. Cambridge University press.

Leduc, T., Bocher, E., González Cortés, F., and Moreau, G. (2009a). GDMS-R: A mixed SQL to manage raster and vector data. In GIS Ostrava 2009 - Symposium on Seamless Geoinformation Technologies, Ostrava, Czech Republic.

Leduc T, Woloszyn P, and Joanne P (2009b). GDMS: A spatial semantics to evaluate soundmarks effects on an urban pedestrian pathway. In $12^{\text {th }}$ AGILE International Conference on Geographic Information Science - AGILE'2009, Hanover, Germany.

Lynch KA (1976). Managing the sense of a region. Cambridge: MIT Press.

Meilinger T, Franz G, and Bülthoff HH (2009). From isovists via mental representations to behaviour: first steps toward closing the causal chain. Environment and Planning B: Planning and Design. Advance online publication.

Morello E and Ratti C (2009). A digital image of the city: 3D isovists in Lynch's urban analysis. Environment and Planning B: Planning and Design, 36(5):837-853.

Peponis J, Wineman J, Rashid M, Hong Kim S, and Bafna S (1997). On the description of shape and spatial configuration inside buildings: convex partitions and their local properties. Environment and Planning B: Planning and Design, 24(5):761-781.

Piombini A (2006). Modélisation des choix d'itinéraires pédestres en milieu urbain approche géographique et paysagère. $\mathrm{PhD}$ thesis, Université de FrancheComté. Ecole Doctorale "langages, espaces, temps, sociétés".

Sarradin F, Siret D, Couprie M, and Teller J (2007). Comparing sky shape skeletons for the analysis of visual dynamics along routes. Planning and design: Environment and planning B, 34(5):840-857.

Stamps AE (2005). Isovists, enclosure, and permeability theory. Environment and Planning B: Planning and Design, 32(5):735-762.

Tandy CRV (1967). The isovist method of landscape survey. Methods of Landscape Analysis. Ed. H C Murray, Landscape Research Group, PO Box 53, Horspath, Oxford, OX33 1WX.

Teller J (2003). A spherical metric for the field-oriented analysis of complex urban open spaces. Planning and design: Environment and planning B, 30(3):339-356. 
Turner A, Doxa M, O’Sullivan D, and Penn A (2001). From isovists to visibility graphs: a methodology for the analysis of architectural space. Planning and design: Environment and planning B, 28(1):103- 121.

Woloszyn, P. and Follut, D. (2000). The visualization of the urban "ambients" parameters. In $14^{\text {th }}$ International symposium "Computer Science for environmental protection" of Gesellschaft für Informatik (GI), pages 173-186, Bonn, Germany. METROPOLIS Verlag.

Woloszyn, P. (2002). From fractal techniques to subjective quantification: towards an urban ambient metric? In Landscape and Architectural Modeling Symposium, pages 1-6, Sousse, Tunisia.

Yang PPJ, Putra SY, and Li W (2007). Viewsphere: a GIS-based 3D visibility analysis for urban design evaluation. Planning and design: Environment and planning B, 34(6):971-992.

Zacharias J (2001). Pedestrian behaviour and perception in urban walking environments. Journal of Planning Literature, 16(1):18. 